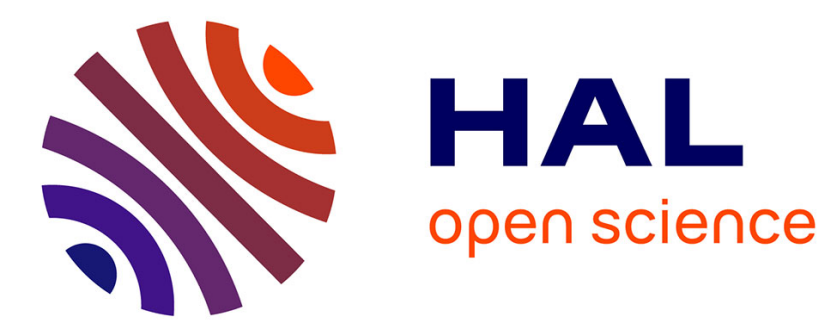

\title{
Compact Antenna Array of Superdirective Elements
}

\author{
Abdullah Haskou, Ala Sharaiha, Sylvain Collardey
}

\section{To cite this version:}

Abdullah Haskou, Ala Sharaiha, Sylvain Collardey. Compact Antenna Array of Superdirective Elements. IEEE Antennas and Wireless Propagation Letters, 2016, 15, pp.1386 - 1389. 10.1109/LAWP.2015.2510382 . hal-01247233

\section{HAL Id: hal-01247233 \\ https://hal.science/hal-01247233}

Submitted on 21 Dec 2015

HAL is a multi-disciplinary open access archive for the deposit and dissemination of scientific research documents, whether they are published or not. The documents may come from teaching and research institutions in France or abroad, or from public or private research centers.
L'archive ouverte pluridisciplinaire HAL, est destinée au dépôt et à la diffusion de documents scientifiques de niveau recherche, publiés ou non, émanant des établissements d'enseignement et de recherche français ou étrangers, des laboratoires publics ou privés. 


\title{
Compact Antenna Array of Superdirective Elements
}

\author{
Abdullah Haskou, Ala Sharaiha, Senior Member, IEEE, and Sylvain Collardey
}

\begin{abstract}
In this letter, we investigate using a two-element parasitic (loaded) superdirective antenna as a unit-element to achieve a compact 3D array. Four elements of a planar parasitic superdirective antenna are integrated in a compact $2 \times 2$ array for UHF band. The array final dimensions are $200 \times 200 \times 70 \mathrm{~mm}^{3}(0.58 \lambda \times 0.58 \lambda \times 0.2 \lambda)$, and it presents a maximum simulated total directivity of $11.4 \mathrm{dBi}$. The antenna dimensions are significantly smaller than classical commercial arrays achieving the same directivity. The measured results are in a good agreement with the simulated ones.
\end{abstract}

Keywords-Antenna array, superdirectivity, PCB, radiation efficiency, directivity

\section{INTRODUCTION}

S OME emerging wireless technologies require accurate beam pointing that can only be achieved using antenna arrays. The main parameter determining the size of an array is the spacing between adjacent radiators, which is typically chosen to be half a wavelength. Reducing the spacing introduces mutual coupling between the elements and, hence, a degradation of the radiation pattern and the gain of the antenna. To make ultimate use of all available degrees of freedom for a given number of radiators, superdirective elements must be taken into consideration, though at the expense of reduced bandwidth and high sensitivity to variations in component values.

Since the early work of I. Uzkov [1], a significant research was done on the design of superdirective arrays [2]-[6]. Early works focused on the design of superdirective 3D wire antennas [2][3]. Two-element supergain Electrically Small Antenna (ESA) arrays were studied in [4]. Later, multiple printed end-fire parasitic superdirective ESAs were presented [5]-[6].

In our previous works we have detailed the design methodology of parasitic superdirective arrays and the tradeoff between the maximum directivity and radiation efficiency in [6]. The integration of two-element electrically small parasitic arrays in PCBs was investigated in [7] where we modify the PCB by including a slot in order to maintain the superdirectivity. Finally, the effect of the excitation cable on measuring PCBmounted arrays was investigated in [8]. In this letter, we investigate using parasitic (loaded) superdirective antennas as unit-elements to realize compact $3 \mathrm{D}$ broadside arrays. A parametric analysis on the inter-element distance demonstrates the necessary tradeoff between the antenna- dimensions, directivity and -radiation efficiency. Although this work is based on two-element array, it can be generalized to N-element arrays. ${ }^{1}$ The rest of the paper is organized as follows: simula-

Manuscript received August 24, 2015; revised November 9, 2015.

The authors are with IETR UMR CNRS 6164- Université de Rennes 1, 35042 Rennes Cedex, France. e-mail: (abdullah.haskou@univ-rennes1.fr).

${ }^{1}$ This work was done with the funding of the French National Research Agency as part of the project "SOCRATE" and the support of the "Images et Reseaux" cluster of Brittany region, France. tion results are presented in section II. Array dimensions effect is studied in section III. Results are validated via measurements in section IV. Finally, section V provides some concluding remarks.

\section{GEOMETRY AND Simulation Results}

\section{A. Unit-Element Design}

The approaches presented in [6], was applied to design a two-element parasitic array integrated in a PCB for $869 \mathrm{M} \mathrm{Hz}$ frequency band. The array is based on an electrically small half-loop antenna printed on a $0.8 \mathrm{~mm}$-thick Rogers RO4003 $\left(\epsilon_{r}=3.55, \tan (\delta)=0.0027\right)$ substrate. Fig. 1(a) shows array geometry and dimensions in millimeters. In this array, exciting the second element and loading the first one with an inductor of $4.3 \mathrm{nH}$, a maximum simulated (HFSS [9]) directivity of $7 d B i$ is achieved as shown in Fig. 1(b). This directivity is $1.8 \mathrm{~dB}$ greater than Harrington's normal directivity limit of an antenna with the same dimensions $(k a=1.08)$, where $a$ is the radius of the smallest sphere enclosing the antenna and $k=2 \pi / \lambda$ is the wave number [10]. The Half Power Beam-Width (HPBW) in horizontal and vertical planes (XOY and YOZ) are respectively $76^{\circ}$ and $108^{\circ}$, and the Front To Back Ratio (FBR) is $6.4 d B$. Furthermore, the radiation pattern does not have any side lobes. Fig. 1(c) shows that antenna's end-fire total directivity $\left(D_{\left(\theta=90^{\circ}, \phi=90^{\circ}\right)}\right)$ is maximal around the resonance frequency of $869 \mathrm{MHz}$. The antenna achieves an impedance bandwidth $\left(S_{11}<-6 d B\right)$ of $2.3 \mathrm{MHz}$ and directivity bandwidth of $\left(D_{\max }-1 d B\right)$ of $4.3 \mathrm{MHz}$. Finally, Fig. 1(d) shows the antenna surface current distribution at the resonance frequency. As it can be noticed, the current on the two elements is on phase opposition which is the condition for having superdirectivity for very small inter-element distances. Due to this current opposition, the antenna presents a relatively low simulated radiation efficiency of $45.6 \%$ (a gain of $3.6 \mathrm{dBi}$ ).

\section{B. 3D Array Design}

Four of the end-fire antenna presented in subsection A are arranged as shown in Fig. 2(a) where the two antennas in the same plane are inverted to increase the distance between the radiating elements. Then, to achieve the maximum directivity in oY direction, this inversion is compensated by a $180^{\circ}$ phase shift in their excitation (elements 1 and 3 are excited out of phase comparing to elements 2 and 4 ). The separating distance (measured between the feeding points) is $152 \mathrm{~mm}$ on $\mathrm{x}$-axis and $200 \mathrm{~mm}$ on $\mathrm{z}$-axis. Hence, the array total dimensions are $200 \times 200 \times 70 \mathrm{~mm}^{3}$. As it can be seen from the antenna simulated 3D total directivity radiation pattern given in Fig. 2(b), the antenna has a directive pattern with a maximum total directivity of $11.4 \mathrm{dBi}$. The HPBW in horizontal and vertical planes are respectively $56^{\circ}$ and $48^{\circ}$, and the FBR is 
$13.2 d B$. The radiation pattern has four side lobes with a Side Lobe Level (SLL) of $-8.3 d B i$. Fig. 2(c) shows that antenna's broadside total directivity $\left(D_{\left(\theta=90^{\circ}, \phi=90^{\circ}\right)}\right)$ is maximal around the resonance frequency of $869 \mathrm{MHz}$ (the input reflection coefficient of the four elements are identical so only one is shown). The antenna has an impedance bandwidth of $1 \mathrm{MHz}$ and a directivity one of $8.7 \mathrm{M} \mathrm{Hz}$. Comparing with the end-fire array, it can be noticed that the array maximum total directivity is increased by $4.4 \mathrm{~dB}$, the horizontal HPBW is divided by 1.4 , and vertical HPBW is divided by 2.3 . The limited improvement in horizontal HPBW is due to the smaller separation in this plane, and hence, a higher mutual coupling as it can be seen in Fig. 2(d).

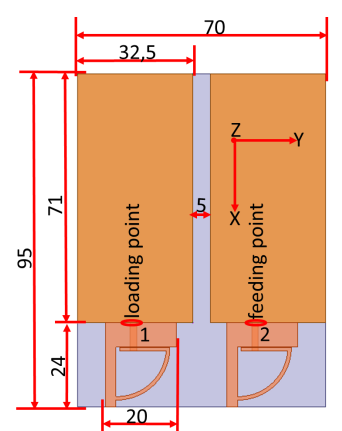

(a)

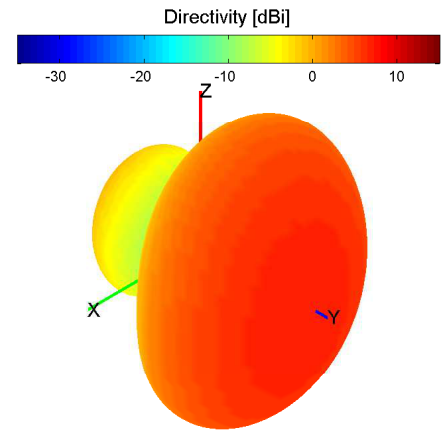

(b)

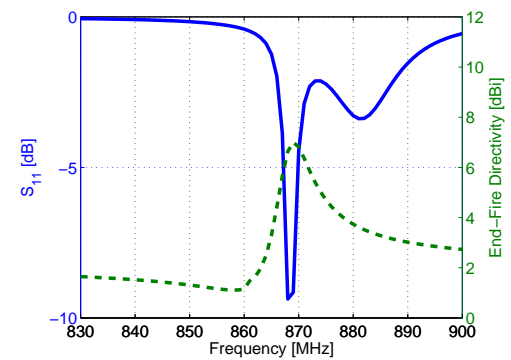

(c)

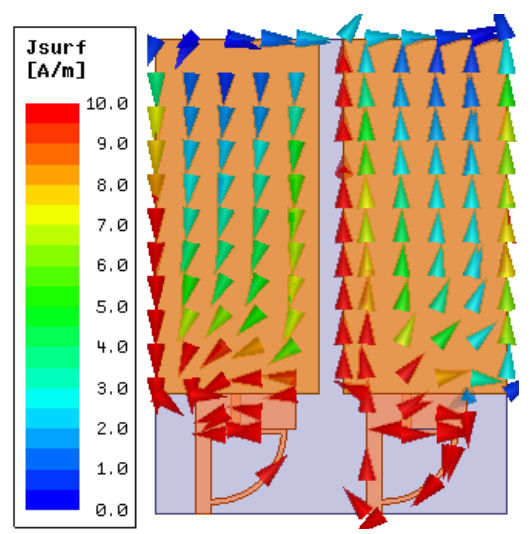

(d)

Fig. 1. End-fire array geometry and simulated parameters. (a) Geometry and dimensions, (b) 3D total directivity radiation pattern, (c) input reflection coefficient magnitude in $\mathrm{dB}$ and broadside directivity and (d) surface current distribution.

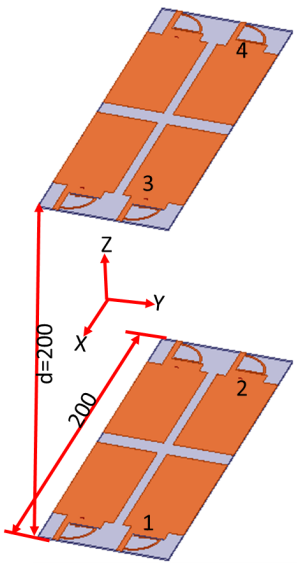

(a)

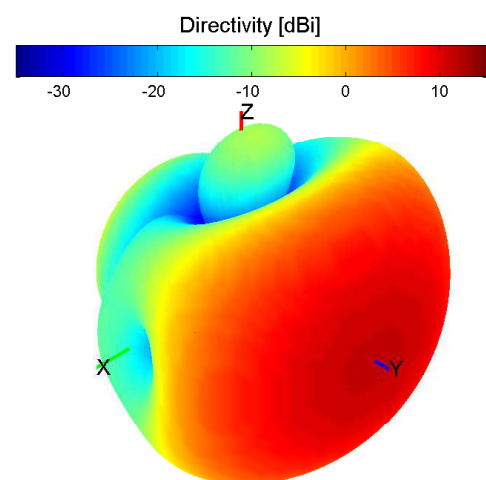

(b)

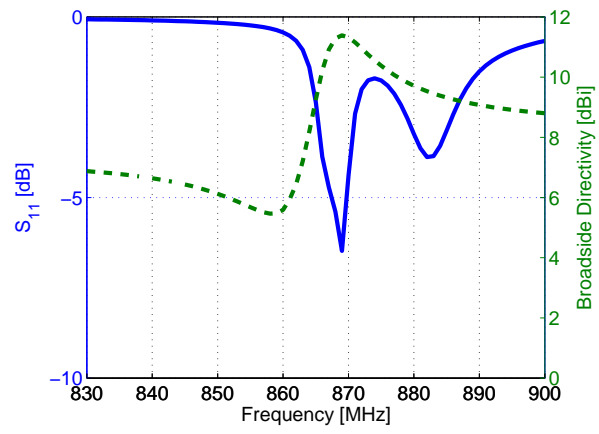

(c)

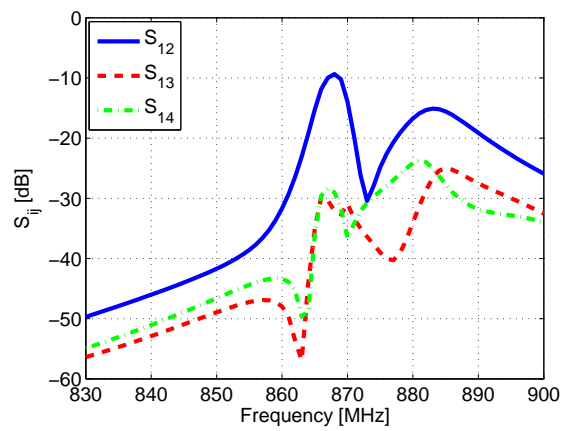

(d)

Fig. 2. Broadside array geometry and simulated parameters. (a) Geometry and dimensions, (b) 3D total directivity radiation pattern, (c) input reflection coefficient magnitude in $\mathrm{dB}$ and broadside directivity and (d) mutual coupling magnitude in $\mathrm{dB}$.

\section{DistANCE EFFECT}

We vary the array distance $(d)$ from $0.01 \lambda$ to $\lambda$ while monitoring the antenna input reflection coefficient, total directivity and radiation efficiency. Fig. 3(a) shows the array simulated input reflection coefficient magnitude in $\mathrm{dB}$ as a function of the distance. The figure shows that for $d=0.01 \lambda$ the array is completely unmatched in the observed frequency band. This is due to the high mutual coupling. As the distance increases the mutual coupling decreases and the array resonance frequency converges to the one of the unit-elements. 
Fig. 3(b) shows the array simulated maximum total directivity and radiation efficiency as a function of the distance. For very small distances, due to the high mutual coupling, the applied loads are not suitable anymore and superdirectivity effect is lost, and hence, the radiation efficiency is maximal. As the distance increases, the superdirectivity effect appears and the radiation efficiency decreases. As expected, we can note that as the distance increases, the achieved directivity increases till $0.7 \lambda$ when it starts decreasing again [11]. Fig. 4 shows array $2 \mathrm{D}$ total directivity radiation patterns in horizontal and vertical planes at the design frequency $(869 \mathrm{M} \mathrm{Hz})$ for several distances. For distances greater than $0.5 \lambda$ side lobes appear in the vertical plane and as the distance increases the SLL also increases, where increasing the distance from $0.5 \lambda$ to $\lambda$ increases the SLL from $-12.8 d B i$ to $6.7 d B i$. We chose to realize the antenna with $d=0.6 \lambda$ due to a constraint on the antenna dimensions.

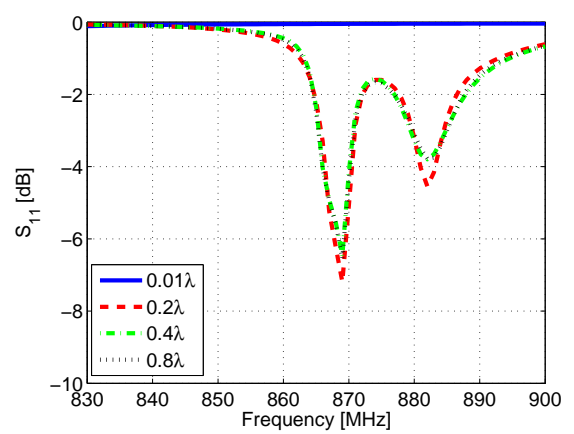

(a)

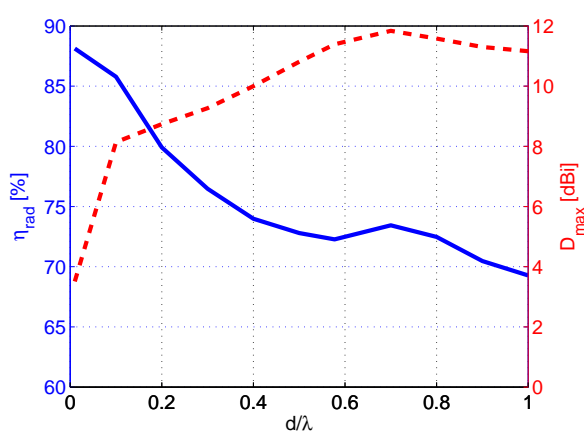

(b)

Fig. 3. Broadside array simulated parameters as a function of the distance (a) Input reflection coefficient magnitude in $\mathrm{dB}$ and (b) total directivity and radiation efficiency.

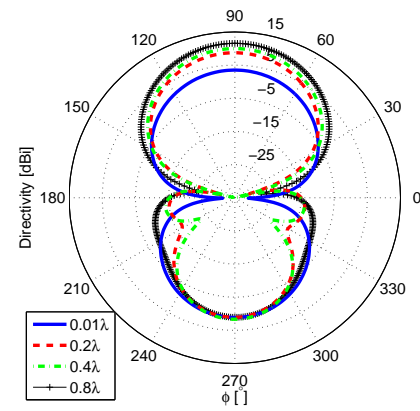

(a)

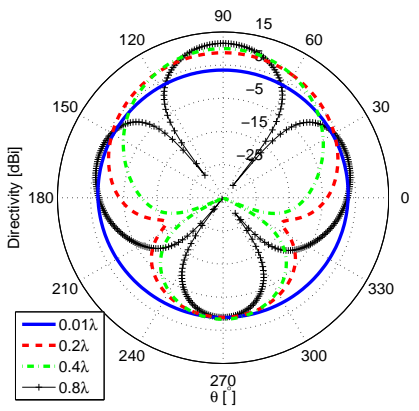

(b)
Fig. 4. Broadside array simulated $2 \mathrm{D}$ total directivity radiation patterns as a function of the distance. (a) Horizontal plane and (b) vertical plane.

\section{Measurement Results}

Fig. 5(a) shows a photograph of the realized prototype of the broadside array. The feeding system is composed of one ZFSCJ-2-4+ power splitter [12], two ZX10-2-20+ power splitter-shifter [13] and four 30 $\mathrm{cm}$-long UFL cables [14]. The antenna far-field radiation pattern was measured in SATIMO stargate (SG 32) near-field measurement system. The measured $3 \mathrm{D}$ total directivity radiation pattern at the resonance frequency is given in Fig. 5(b). The figure shows a maximum total directivity of $11.3 d B i$ in the broadside direction (towards oY). Fig. 5(c) shows measured input reflection coefficient magnitude for the unit-elements and the antenna with the feeding system as well as the maximum total directivity versus frequency. The measured resonance frequency of the unit-elements is around $883 \mathrm{MHz}$ (a frequency shift of $1.8 \%$ ). This shift is probably due to the antenna environment (UFL cable effect, SMA connector and the dispersion of the commercial SMD loads). We can note that the feeding system presents an approximate insertion loss of $3.5 d B$ at the resonance frequency. This loss is distributed as follows: $1.5 d B$ in the splitter, $0.6 d B$ in the splitter-shifter, $1 d B$ in the UFL cable and $0.4 d B$ in the connectors. The antenna (without the feeding system) has an impedance bandwidth of $1.9 \mathrm{MHz}$. The figure also shows that the antenna directivity is maximal around the resonance frequency with a directivity bandwidth of $13.5 \mathrm{MHz}$. Fig. 6(a) and Fig. 6(b) show measured 2D total directivity radiation patterns. The HPBW in both horizontal and vertical planes are $56^{\circ}$, the FBR is $12.4 d B$ and SLL is $-2.6 d B i$. The measured pattern is in a very good agreement with the simulated one in the main-beam direction. The small difference in the backward direction may be attributed to the measuring system and environment. The antenna radiation efficiency measured in a reverberation chamber [15], after compensating the losses in the feeding system, is about $58 \%$, and hence. the antenna measured gain is $8.9 \mathrm{dBi}$. 


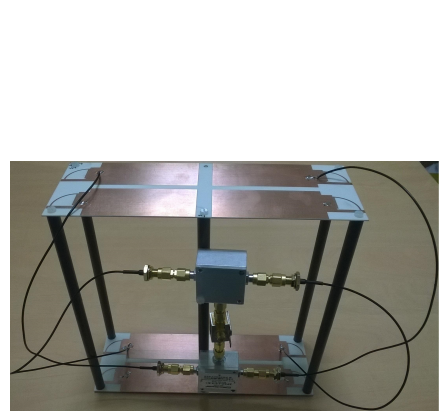

(a)

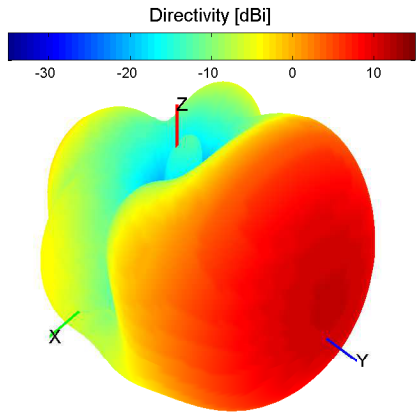

(b)

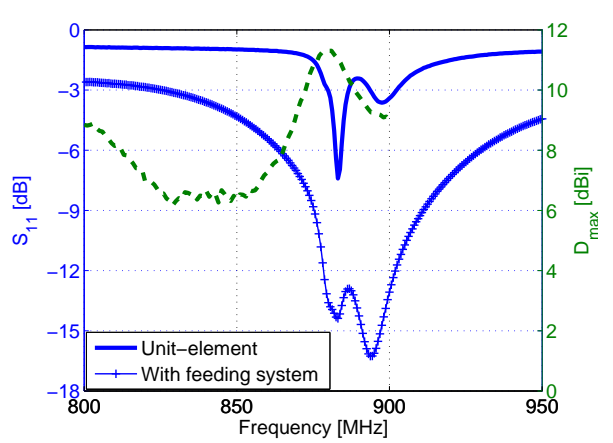

(c)

Fig. 5. Broadside array prototype and measured results. (a) Fabricated prototype, (b) 3D total directivity radiation pattern and (c) input reflection coefficient magnitude in $\mathrm{dB}$ and maximum total directivity.

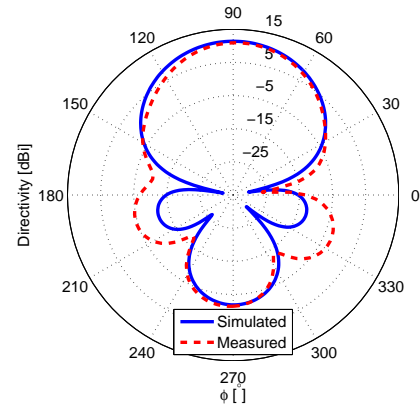

(a)

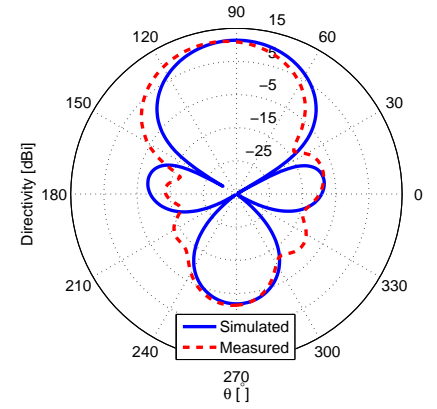

(b)
Fig. 6. Broadside array measured 2D total directivity radiation pattern.(a) Horizontal plane and (b) vertical plane.

\section{CONClusion}

In this letter, a new strategy is presented to obtain a 3D small-size broadside array using compact end-fire unitelements for UHF band. The array total dimensions were $0.58 \lambda \times 0.58 \lambda \times 0.2 \lambda$ and it achieved a total directivity of $11.4 d B i$. The measured results were in a very good agreement with the simulated ones. This array is significantly compact compared with others achieving such directivities. Although this work is based on a two-element array, it can be generalized on N-element arrays while keeping in mind the tradeoff between the array- dimensions, -directivity and -radiation efficiency.

\section{REFERENCES}

[1] I. Uzkov, "An Approach to the Problem of Optimum Directive Antennae Design", C. R. (Doklady) Acad. Sci. l'URSS, vol. 53, no. 1, pp. 35-38, 1946.

[2] E. E. Altshuler, T. H. O’Donnell, A.D. Yaghjian, and S. R. Best, "A Monopole Superdirective Array", IEEE Trans. Antennas Propag., vol. 53, no. 8, pp. 2653-2661, Aug. 2005.

[3] T. H. O’Donnell, and A. D. Yaghjian, "Electrically Small Superdirective Arrays Using Parasitic Elements", in Proc. IEEE Antennas Propag. Soc. Int. Symp., pp. 3111,3114, 9-14 Jul. 2006.

[4] A. D. Yaghjian, T. H. O’Donnell, E. E. Altshuler, and S. R. Best "Electrically Small Supergain End-Fire Arrays", Radio Sci., vol. 43, 2008.

[5] P. Sharma, D. Arora, and H. Gupta, "Designing Superdirective Patch Antenna Array Using Metamaterial", Int. J. of Engineering Research \& Tech. (IJERT), vol. 1, Issue 8, Oct. 2012.

[6] A. Haskou, A. Sharaiha, and S. Collardey, "Design of Small Parasitic Loaded Superdirective End-Fire Antenna Arrays", IEEE Trans. Antennas Propag., vol. 63, no. 12, pp. 5456-5464, Dec. 2015.

[7] A. Haskou, A. Sharaiha, and S. Collardey, "Integrating Superdirective Electrically Small Antenna Arrays in PCBs", IEEE Antennas and Wireless Propag. Lett., doi: 10.1109/LAWP.2015.2425913.

[8] A. Haskou, S. Collardey, and A. Sharaiha, "Measuring Superdirective Electrically Small Antenna Arrays Mounted on PCBs", Microwave and Optical Tech. Lett., vol. 57, Issue 10, pp. 2269-2274, Oct. 2015.

[9] ANSYS HFSS, Pittsburg, PA 15219, USA.

[10] R. F. Harrington, "On the Gain and Beamwidth of Directional Antennas", IRE Trans Antennas Propag., pp. 219-225, Jul. 1958.

[11] R. C. Hansen, and R. E. Collin, "Small Antenna Handbook", John Wiley \& Sons Inc., Hoboken, New Jersey, 2011.

[12] www.minicircuits.com/pdfs/ZFSCJ-2-4.pdf

[13] www.minicircuits.com/pdfs/ZX10-2-20.pdf

[14] http://docs-europe.electrocomponents.com/webdocs/12a7/0900766b812a766a.pdf

[15] G. Le Fur, C. Lemoine, P. Besnier, A. Sharaiha, "Performances of UWB Wheeler Cap and Reverberation Chamber to Carry Out Efficiency Measurements of Narrowband Antennas", IEEE Antennas and Wireless Propag. Lett., vol. 8, pp. 332,335, 2009. 